\title{
RECOMENDAÇÃO PERSONALIZADA DE CONTEÚDO PARA SUPORTE À APRENDIZAGEM INFORMAL NO CONTEXTO DA SAÚDE
}

\author{
Alisson Alan Lima da Costa ${ }^{1}$ \\ Francisco Milton Mendes Neto ${ }^{1}$ \\ Enio Lopes Sombra ${ }^{1}$ \\ Jonathan Darlan Cunegundes Moreira ${ }^{1}$ \\ Ricardo Alexsandro de Medeiros Valentim² \\ Antonio Farias Alves Sales ${ }^{3}$ \\ ${ }^{1}$ Programa de Pós-Graduação em Ciência da Computação - PPGCC, Universidade Federal \\ Rural do Semi-Árido - UFERSA, Mossoró, Rio Grande do Norte, Brasil. \\ ${ }^{2}$ Universidade Federal do Rio Grande do Norte - UFRN, Natal, Rio Grande do Norte, Brasil. \\ ${ }^{3}$ Núcleo de Tecnologia em Engenharia de Software - NTES, Universidade Federal Rural do \\ Semi-Árido - UFERSA, Mossoró, Rio Grande do Norte, Brasil. \\ \{alisson, miltonmendes, enio, jdarlan\}@ufersa.edu.br; ricardo.valentim@ufrnet.br; \\ antonio.fazsa@gmail.com
}

Resumo. Pessoas com doenças crônicas sofrem com limitações impostas por sua condição de saúde e aprender mais sobre a doença ajuda na melhoria de sua qualidade de vida. O uso em massa dos dispositivos móveis e o advento das ferramentas da web 2.0 contribuíram para o surgimento do conceito de saúde 2.0, que disponibiliza meios de promover a aprendizagem informal através da recomendação personalizada de conteúdo aos usuários, indicando conteúdos relevantes relacionados à sua condição de saúde. Desta forma, conhecendo-se o perfil do usuário e aplicando mecanismos de recomendação de conteúdo com o uso de algoritmo genético, é possível, saber a semelhança entre usuários e conteúdos e recomendar conteúdos de seu interesse. Diante disso, este artigo propõe um sistema de recomendação personalizada de conteúdos, como parte do modelo MobiLEHealth, para prover a aprendizagem informal no contexto da saúde, apoiando pessoas com doenças crônicas.

Palavras-chaves: sistema de recomendação personalizada, aprendizagem informal, saúde 2.0, algoritmo genético.

\section{PERSONALIZED RECOMMENDATION OF CONTENT FOR SUPPORTING INFORMAL LEARNING IN THE HEALTH CONTEXT}

\begin{abstract}
People with chronic diseases suffer from limitations imposed by their health condition and learn more about the disease help in improving their quality of life. The mass use of mobile devices and the advent of web 2.0 tools contributed for the emergence of the concept of Health 2.0, which provides means to promote informal learning through personalized content recommendation to the users, indicating relevant content related to their health condition. Thus, knowing the user profile and applying recommendation engines of content using genetic algorithm is possible, discover the similarity among users and the contents, recommend content of their interest. Therefore, this paper proposes a system for personalized content recommendation, as part of the model MobiLEHealth, to provide informal learning in the context of health, supporting people with chronic diseases.
\end{abstract}

Keywords: Personalized Recommendation Systems, Informal Learning, Health 2.0, Genetic Algorithms. 


\section{Introdução}

As doenças crônicas representam um alto percentual de mortalidade no mundo. Porém, boa parte delas pode ser prevenida e controlada, promovendo uma melhor qualidade de vida. Para tanto, é preciso conhecer a doença e, em seguida, fazer o tratamento recomendado (com medicamentos, dietas, hábitos saudáveis, mudanças no estilo de vida e prática de exercícios). Porém o sucesso do tratamento só acontece através da aceitação pelo paciente. Nisto, o conceito de saúde 2.0 torna-se um fator motivador, pois une aprendizagem informal ao uso de tecnologias e ferramentas da web 2.0 (redes sociais, registros pessoais de saúde, blogs, vídeos, etc.) como forma de capturar, através da experiência do usuário, informações referentes à saúde e, pela combinação desses dados e informações, fornecer cuidados e conteúdos personalizados (Hughes et al., 2008).

Diante deste cenário, este artigo apresenta um sistema para recomendações personalizadas de conteúdos como parte de um modelo para apoiar a aprendizagem informal no contexto de Saúde 2.0, denominado MobiLEHealth (Mendes Neto et al., 2014a, b). Com foco na aprendizagem informal, o sistema proposto, é direcionado às pessoas com doença crônica e em tratamento domiciliar, promovendo o conhecimento sobre a doença e, consequentemente, uma melhoria na sua qualidade de vida.

No MobiLEHealth, as recomendações personalizadas de conteúdos, que são geradas no intuito de prover a aprendizagem informal, são baseadas em um perfil do usuário, que é enriquecido a partir de dados coletados da interação deste com uma interface personalizada, por meio de ferramentas da web 2.0.

\section{Referencial Teórico}

\subsection{Aprendizagem Informal}

Na aprendizagem informal, o indivíduo é responsável pelo seu aprendizado, através de informações cotidianas do seu ambiente (Jiugen et al., 2011), e tem como principais características (i) a autonomia, onde o indivíduo é responsável por sua aprendizagem e determina o objetivo a ser alcançado, o conteúdo a ser abordado e como este será processado; (ii) o conhecimento, obtido por meio das interações sociais e profissionais; (iii) a diversidade, obtida através de experiências diárias, recursos do ambiente, bibliotecas, redes sociais, dentre outros.

As tecnologias móveis ajudam no processo de aprendizagem informal através de um ambiente ubíquo favorecendo a autoaprendizagem, relações sociais, o compartilhamento de experiências e a aprendizagem colaborativa, proporcionando o acesso ao conhecimento, em qualquer lugar e a qualquer momento.

\subsection{Algoritmo Genético}

Algoritmo Genético (AG) pode ser definido como uma metáfora do processo biológico de evolução natural. Eles são responsáveis por tentar encontrar uma solução para os problemas que não têm algoritmos conhecidos. Em geral, o AG toma como entrada uma população inicial e os indivíduos mais adequados são selecionados para a solução do problema, de acordo com os critérios de avaliação. Se os indivíduos selecionados não são os melhores, é feita uma nova combinação (Rothlauf, 2006).

A execução de um AG pode ser resumida nos seguintes passos: a) Inicializa-se a população de cromossomos; b) Avalia-se cada cromossomo da população; c) Seleciona- 
se os indivíduos que irão assumir o papel de pais para gerar novos cromossomos; d) Aplica-se as operações de recombinação e mutação, a fim de selecionar os indivíduos como pais, criando uma nova geração; e) Elimina-se os cromossomos da geração anterior; f) Avalia-se os cromossomos que foram gerados e inseridos na população; g) Se os cromossomos encontrados representam a solução esperada para o problema ou o número máximo de gerações foi alcançado, ou o AG não conseguiu mostrar mais progresso, a execução é terminada. Caso contrário, a execução retorna para o passo c.

\subsection{Sistemas de Recomendação}

O aumento de meios de disponibilização de conteúdo, podendo ser serviços ou informação, através de sistemas web, provoca uma situação onde o usuário possui muitas opções de escolha antes mesmo de estar apto a selecionar uma opção que atenda suas necessidades. Os Sistemas de Recomendação (SRs) buscam amenizar os impactos gerados por essa sobrecarga de informação. Ao contrário de sistemas de busca, SRs permitem o acesso à informação sem uma consulta explícita de um dado usuário (Coello et al., 2013).

Para tanto, os SRs utilizam repositórios de informação e dados de preferência dos usuários para direcionar conteúdos aos indivíduos com potenciais interesses. Um dos desafios dos SRs é realizar a indicação de produtos, serviços e/ou informação que melhor atendam às expectativas dos usuários, bem como ao seu perfil.

Segundo Vieira e Nunes (2012), os SRs podem ser classificados em três tipos: (i) Sistema de Recomendação Baseada em Conteúdo (SRBC) que é realizada através de informações recuperadas do usuário (perfil, comportamento, etc.) e/ou do seu histórico de escolhas; (ii) Sistema de Recomendação Colaborativa (SRC) que leva em consideração as escolhas realizadas por usuários com características similares e (iii) Sistema de Recomendação Híbrida (SRH) que efetua a recomendação através das técnicas utilizadas pelos SRBC e SRC, de forma a atingir um maior número de possibilidades e sugerir objetos que diretamente não aparentam estar relacionados (Martins Júnior et al., 2011).

\section{Trabalhos Relacionados}

A utilização de SRs tem sido bastante útil para o usuário na busca por informações de seu interesse na web. No âmbito da aprendizagem informal, os usuários são expostos a uma grande quantidade de recursos de informação, onde muitas vezes não estão aptos para realizar tais escolhas. Desta forma, um SR vem a suprir esta carência recomendando recursos apropriados e auxiliando na escolha do usuário, que passará a lidar com um volume menor de informação.

Na literatura existem diversos trabalhos e projetos relacionados à aplicação de SRs com foco em aprendizagem informal na área da saúde. Em Childs (2004), é apresentado o projeto “Judge”, que tem o objetivo de desenvolver diretrizes de qualidade de sites web na área de saúde em relação ao uso de medicamentos. Já em Su e Wu (2011), é proposto um SR voltado para blogs na área da saúde, onde são observados sites de redes sociais, como Twitter, Facebook, Linkedln, entre outros.

Este trabalho propõe um Sistema de Recomendação Híbrido Ubíquo (SRHU) que tem como diferencial a combinação de diferentes técnicas de recomendação e Algoritmo Genético (AG), para prover a aprendizagem informal através de recomendação de conteúdos relacionados à saúde de acordo com o perfil dos usuários. 


\section{Sistema de Recomendação Proposto}

A solução proposta consiste de um sistema de recomendação que envolve a Recomendação Colaborativa e a Recomendação Baseada em Conteúdo. Além disso, ainda considera o Enriquecimento Semântico do perfil do usuário e do conteúdo, e também o contexto do usuário no momento da recomendação, implementando dessa forma, o que chamamos de Sistema de Recomendação Híbrida Ubíqua (SRHU).

O SRHU tem como principais funções: i) Analisar periodicamente a base de dados e o Sistema de Enriquecimento Semântico (SES) gerando pré-listas de conteúdos aptos à recomendação, sendo estas a Lista de Colaboradores (LC), a Lista Colaborativa Ponderada (LCP) e a Lista Baseada em Conteúdo (LBC); e ii) Com as pré-listas obtidas gera-se a Lista Híbrida Ubíqua (LHU) e por fim a Recomendação Conjunta.

\subsection{Recomendação Colaborativa Ponderada}

A Recomendação Colaborativa consiste na análise das escolhas e avaliações, para conteúdo, de usuários semelhantes ao usuário alvo. Nesta recomendação obtém-se uma lista de usuários semelhantes ao usuário alvo, denominada de Lista de Colaboradores, e, através das relações entre usuários e conteúdos, gera-se a Lista Colaborativa Ponderada.

\subsubsection{Lista de Colaboradores}

É onde são obtidos os usuários com maior grau de semelhança, ordenando-os e vinculando-os ao Índice de Semelhança entre Usuários - ISU. Neste, o cálculo é feito sob os aspectos: (i) Metadados (S1): Analisa os dados (idade, sexo, religião, cor/raça, doença crônica) de ambos os usuários; (ii) Relações (S2): As relações existentes entre usuários: amigos, requisitou amizade, amizade requisitada, rejeitou amizade e amizade rejeitada; (iii) Conteúdos (S3): Analisa quais relações com conteúdo são comuns aos usuários alvo, como: aceitação e rejeição de recomendação e a visualização de conteúdo; (iv) Locais (S4): Analisa quais relações com locais são comuns aos usuários alvo, como: aceitação e rejeição de recomendação, e de visualização de conteúdo; (v) Frequência (S5): São os histogramas de frequência que contêm as respostas de aceitação e rejeição nas recomendações geradas para os usuários, conteúdos e locais; (vi) Tags (S6): Analisa a semelhança entre a lista de tags dos usuários alvo; (vii) Enriquecimento Semântico (S7): Analisa e obtém a semelhança entre os enriquecimentos semânticos dos usuários alvo, comparando as afinidades de dois usuários com cada domínio.

O ISU entre o usuário “i” e o usuário “j” é calculado como sendo a média ponderada de cada uma das semelhanças já citadas. No ISU (Equação 1), o $S_{\mathrm{i}}$ é o nível de semelhança de uma característica e $\mathrm{P}_{\mathrm{i}}$ é o peso dessa característica na avaliação.

$$
\mathrm{ISU}_{\mathrm{i}, j}=\frac{\mathrm{S} 1 * \mathrm{P} 1+\mathrm{S} 2 * \mathrm{P} 2+\mathrm{S} 3 * \mathrm{P} 3+\mathrm{S} 4 * \mathrm{P} 4+\mathrm{S} 5 * \mathrm{P} 5+\mathrm{S} 6 * \mathrm{P} 6+\mathrm{S} 7 * \mathrm{P} 7}{\mathrm{P} 1+\mathrm{P} 2+\mathrm{P} 3+\mathrm{P} 4+\mathrm{P} 5+\mathrm{P} 6+\mathrm{P} 7}
$$

Equação 1 - Cálculo do Índice de Semelhança de Usuários - ISU

\subsubsection{Lista Colaborativa Ponderada}

A Lista Colaborativa Ponderada (LCP) é objeto da análise do histórico das relações com conteúdo dos usuários presentes na Lista de Colaboradores de cada usuário alvo. O SRHU busca na Lista de Colaboradores de cada usuário, as relações destes com os conteúdos e suas respectivas avaliações. Através disto, obtém-se o Índice de Semelhança Colaborativa - ISC, necessário para realizar o cálculo da LCP. 
O ISC i,k $_{\text {de }}$ um determinado conteúdo "k" com um usuário “i” é calculado como sendo a média de todas as avaliações deste conteúdo pelos n usuários presentes na Lista Colaborativa do usuário i, ponderada por seus respectivos $\operatorname{ISU}_{\mathrm{i}, \mathrm{j}}$. Seu resultado é usado no cálculo da Lista Ubíqua e da Lista Híbrida. A Equação 2 mostra a fórmula do ISC.

$$
\operatorname{ISC}_{i, k}=\frac{1}{t} \sum_{j=0}^{n-1} A_{j, k *} \operatorname{ISU}_{i, j}
$$

Equação 2 - Cálculo do Índice de Semelhança de Conteúdo - ISC

\subsection{3. Índice de Semelhança Semântica entre Usuários}

Este índice é obtido com base no resultado do cálculo do ISU (Subseção 4.1.1.), onde o índice S7 é calculado como resultado do retorno do SES. Como o SES calcula apenas o quanto um usuário se interessa por um determinado domínio, para calcularmos o quanto dois usuários, “i” e “j” se assemelham semanticamente, definimos a Equação 3.

$$
\mathrm{S}_{\mathrm{i}, \mathrm{j}}=\frac{10 * \mathrm{n}-\sum_{\mathrm{k}=1}^{\mathrm{n}}\left|\mathrm{A}_{\mathrm{i}, \mathrm{k}}-\mathrm{A}_{\mathrm{j}, \mathrm{k}}\right|}{\mathrm{n}}
$$

Equação 3 - Cálculo do Indice de Semelnança Semântica entre Usuários

\subsection{Recomendação Baseada em Conteúdo}

A Recomendação Baseada em Conteúdo (RBC) é executada em toda a base de usuários gerando, para cada um deles, uma Lista Baseada em Conteúdo (LBC). Ela calcula o Índice Baseado em Conteúdo (IBC) que representa o quanto um conteúdo é recomendável a um usuário, analisando os históricos do conteúdo e do usuário.

O IBC é calculado sob os seguintes aspectos: (i) Metadados (S1): Analisa os dados do histórico de visualização de conteúdo do usuário, como tipo, tamanho, duração, autor e tempo da publicação; (ii) Tags (S2): Analisa a semelhança entre a lista de tags do usuário e do conteúdo; (iii) Locais (S3): Analisa quais relações com locais são comuns ao usuário e ao conteúdo. As relações são: aceitação ou rejeição de recomendação, e de visualização de conteúdo; (iv) Frequência (S4): Refere-se aos histogramas de frequência, citados na Subseção 4.1.1; (v) Enriquecimento Semântico (S5): Analisa a semelhança entre os enriquecimentos semânticos dos usuários.

O IBC entre o usuário “i” e o usuário “j” é calculado como a média ponderada de cada uma das semelhanças citadas acima. O IBC é obtido pela Equação 4, onde $S_{\mathrm{i}}$ é o nível de semelhança de determinada característica e $\mathrm{P}_{\mathrm{i}}$ é o peso dessa característica na avaliação. Os n maiores resultados de IBC, aliados aos respectivos pares, usuário e conteúdo, são inseridos na Lista Baseada em Conteúdo de tamanho n de um usuário.

$$
\mathrm{IBC}_{\mathrm{i}, j}=\frac{\mathrm{S} 1 * \mathrm{P} 1+\mathrm{S} 2 * \mathrm{P} 2+\mathrm{S} 3 * \mathrm{P} 3+\mathrm{S} 4 * \mathrm{P} 4+\mathrm{S} 5 * \mathrm{P} 5}{\mathrm{P} 1+\mathrm{P} 2+\mathrm{P} 3+\mathrm{P} 4+\mathrm{P} 5}
$$

Equação 4 - Cálculo do Índice Baseado em Conteúdo - IBC

\subsection{1. Índice de Semelhança entre Usuário e Conteúdo}

Neste índice, o cálculo é feito para saber o quanto um conteúdo se relaciona com um determinado domínio. A semelhança semântica de um usuário com um conteúdo é obtida através da Equação 5. Onde, $S 5_{i, j}$ é o índice de semelhança semântica entre o usuário i e o conteúdo j; n é o número de domínios inseridos no SES; $A_{i, k}$ é o índice de interesse do usuário i pelo domínio k, retornado pelo SES; e $\mathrm{B}_{\mathrm{j}, \mathrm{k}}$ é o índice que mede quanto o conteúdo j se relaciona com o domínio k, retornado pelo SES. 


$$
S 5_{i, j}=\frac{10 * \mathrm{n}-\sum_{k=1}^{n}\left|A_{i, k}-B_{j, k}\right|}{n}
$$

Equação 5 - Cálculo do Índice de Semelhança Semântica entre Usuário e Conteúdo.

\subsection{Recomendação Baseada na Ubiquidade}

A Recomendação Baseada na Ubiquidade (RBU) é executada em tempo real, para um usuário alvo, e calcula o Índice de Recomendação Ubíquo (IRU), que mostra a adequação de um conteúdo ao contexto do usuário. A RBU é executada apenas sobre as Listas Baseada em Conteúdo e Colaborativa Ponderada, garantindo a resposta em tempo hábil ao contexto do usuário e um novo índice aos conteúdos dessas listas, o IRU.

O cálculo do IRU é feito sob os seguintes aspectos: (i) Frequência do Conteúdo (S1): Analisa os histogramas de aceitação, rejeição e visualização do conteúdo em relação ao horário no contexto do usuário alvo; (ii) Histórico de Locais do Conteúdo (S2): Analisa o histórico de relações com locais do conteúdo e sua adequação ao local considerado no contexto do usuário alvo; (iii) Situação do Usuário (S3): Analisa a adequação do conteúdo em relação à velocidade de locomoção do usuário alvo; (iv) Situação do Dispositivo (S4): Analisa o dispositivo (percentual de bateria e velocidade da conexão com a internet) e sua adequação ao contexto para visualizar o conteúdo.

O IRU do conteúdo “j” em relação ao contexto é obtido como a média ponderada de cada uma das semelhanças citadas. Na Equação 6, temos: $S_{i}$ que é o nível de semelhança de determinada característica e $\mathrm{P}_{\mathrm{i}}$ é o peso dessa característica na avaliação. Os melhores resultados são gravados na Lista Baseada na Ubiquidade (LBU).

$$
\mathrm{IRU}_{\mathrm{j}}=\frac{\mathrm{S} 1 * \mathrm{P} 1+\mathrm{S} 2 * \mathrm{P} 2+\mathrm{S} 3 * \mathrm{P} 3+\mathrm{S} 4 * \mathrm{P} 4}{\mathrm{P} 1+\mathrm{P} 2+\mathrm{P} 3+\mathrm{P} 4}
$$

Equação 6 - Cálculo do Índice de Recomendação Ubíqua - IRU

\subsection{Recomendação Híbrida}

A Recomendação Híbrida Ubíqua (RHU) é executada logo após a geração da LBU para um determinado usuário alvo, e calcula o Índice de Recomendação Híbrida (IRH). A RHU gera a Lista Híbrida Ubíqua (LHU) que é uma lista de conteúdos ligados aos seus respectivos IRHs, os quais são resultantes da análise da Lista Colaborativa Ponderada, da Lista Baseada em Conteúdo e da Lista Baseada na Ubiquidade. O IRH do conteúdo “j” para o usuário "i” considerando seu contexto é calculado como a média ponderada de cada um dos índices das listas descritas anteriormente, de acordo com a Equação 7.

$$
\mathrm{IRH}_{\mathrm{i}, \mathrm{j}}=\frac{\mathrm{ICP} * \mathrm{P} 1+\mathrm{IBC} * \mathrm{P} 2+\mathrm{IBU} * \mathrm{P} 3}{\mathrm{P} 1+\mathrm{P} 2+\mathrm{P} 3}
$$

Equação 7 - Cálculo do Índice de Recomendação Híbrido - IRH

Os valores dos pesos P1, P2 e P3 definem o quanto a recomendação é mais colaborativa, baseada em conteúdo ou sensível ao contexto (ubíqua). Tais pesos variam conforme as ações do usuário (aceitação ou rejeição) em cada recomendação. Na primeira recomendação de um usuário, seus respectivos pesos são iguais a 5,0. O número de recomendações (NR) respondidas pelo usuário é armazenado e serve para o cálculo da adequação dos pesos, que ocorre conforme as Equações 8, 9 e 10.

$$
\mathrm{P} 1=\frac{\mathrm{P} 1 * \mathrm{NR}+\mathrm{k} * \mathrm{ICP}}{\mathrm{NR}+1}
$$

Equação 8 - Cálculo do Peso da Recomendação Colaborativa 


$$
\mathrm{P} 2=\frac{\mathrm{P} 2 * \mathrm{NR}+\mathrm{k} * \mathrm{IBC}}{\mathrm{NR}+1}
$$

Equação 9 - Cálculo do Peso da Recomendação Baseada em Conteúdo

$$
\mathrm{P} 3=\frac{\mathrm{P} 3 * \mathrm{NR}+\mathrm{k} * \mathrm{IBU}}{\mathrm{NR}+1}
$$

\section{Equação 10 - Cálculo do Peso da Recomendação Ubíqua}

Nos cálculos acima, o valor de k varia de 1 (se a recomendação for aceita) até -1 (caso a recomendação seja rejeitada). Após a ação de aceitação ou rejeição do usuário alvo, o valor de NR é incrementado em 1 . Assim, os pesos se ajustam às preferências de cada usuário, personalizando assim as futuras recomendações para os mesmos.

\subsection{Recomendação Conjunta}

A Recomendação Conjunta utiliza-se de técnicas de formação de grupos, priorizando assim que elementos da recomendação final não disputem pelas mesmas características do usuário. Isso é feito após a análise de todas as possibilidades de combinações na lista de recomendação híbrida. A partir da Lista Híbrida Ubíqua que possui n conteúdos e se deseja fazer uma recomendação de p conteúdos, o número $\mathrm{N}$ de combinações a serem analisadas é obtido como apresentado na Equação 11.

$$
\mathrm{N}=\frac{\mathrm{n} !}{\mathrm{p} !(\mathrm{n}-\mathrm{p}) !}
$$

\section{Equação 11 - Cálculo do Número de Recomendações Possíveis}

A complexidade da análise de todas as possibilidades cresce de forma fatorial, o que justifica o uso de uma técnica aproximativa, no caso Algoritmo Genético (AG), para a escolha da melhor recomendação conjunta. No exemplo apresentado nas Tabelas 1 e 2, dada a LHU de conteúdos de tamanho $n=120$ a serem recomendados, teremos uma recomendação conjunta de tamanho $p=5$.

Tabela 1 - Lista Híbrida Ubíqua

\begin{tabular}{|c|c|c|c|c|c|c|}
\hline $\begin{array}{c}\text { Conteúdo } \\
1\end{array}$ & $\begin{array}{c}\text { Conteúdo } \\
2\end{array}$ & $\begin{array}{c}\text { Conteúdo } \\
3\end{array}$ & $\begin{array}{c}\text { Conteúdo } \\
4\end{array}$ & $\begin{array}{c}\text { Conteúdo } \\
\cdots\end{array}$ & $\begin{array}{c}\text { Conteúdo } \\
120\end{array}$ \\
\hline IRH=9,80 & IRH=9,25 & IRH=9,48 & IRH=9,60 & IRH=8,92 & $\cdots$ & IRH=5,90 \\
\hline
\end{tabular}

Tabela 2 - Recomendação Conjunta (cromossomo melhor avaliado pela função fitness)

\begin{tabular}{|l|l|l|l|l|}
\hline Conteúdo 16 & Conteúdo 25 & Conteúdo 76 & Conteúdo 1 & Conteúdo 9 \\
\hline IRH=8,87 & IRH=9,15 & IRH=7,38 & IRH=8,91 & IRH=9,03 \\
\hline
\end{tabular}

O resultado da recomendação conjunta foi obtido através da escolha entre os melhores IRHs da Lista Híbrida Ubíqua e com base na análise feita considerando-se a melhor avaliação do cromossomo pela função fitness, que teve como critérios a soma dos tempos, repetições de autores, subtipo, e defeitos genéticos (conteúdos repetidos).

A função fitness prioriza principalmente a diversidade, evitando que na lista final apareçam autores repetidos, ou apenas um subtipo de conteúdo (vídeo, texto ou áudio) ou mesmo que a soma dos tempos totais ultrapassem a quantidade do que é característico do usuário aceitar em um determinado intervalo de tempo. Dessa forma, objetiva-se alcançar uma maior aceitação das recomendações realizadas.

O modelo de AG desenvolvido possui as seguintes características: (a) Gene: Cada gene é representado por uma recomendação de conteúdo a um usuário alvo, 
escolhidos a partir da LHU; (b) Cromossomo (indivíduo): É um conjunto de genes que representam uma possível recomendação conjunta; (c) População Inicial: É gerada a partir da LHU, escolhendo-se aleatoriamente, recomendações de conteúdo que serão os genes que formarão cada indivíduo; (d) Função Fitness: Avalia um cromossomo ou indivíduo, com base nos critérios de IRH, evitando características conflitantes (subtipo, autor, tempo), e reduzindo a avaliação dos cromossomos com defeitos genéticos (genes repetidos); (e) Cruzamento: Onde foi utilizado o conceito de dominância entre os genes, onde um gene (recomendação) com maior rating será dominante sobre um de menor; (f) Seleção: Teve os critérios de seleção com base em Estado Estacionário e Elitismo, onde metade da população (melhor avaliada) sobrevive e gera mais uma metade de população nova (nova geração); (g) Mutação: Ocorre pela troca de posições de genes de um mesmo cromossomo, evitando assim a amarração em falsos máximo da função fitness.

\section{Validação}

A validação conta com dois domínios (Diabetes e Esclerose Lateral Amiotrófica - ELA) e com a criação de dados fictícios de instâncias das classes Person, Location e Content. A inserção de dados nas relações e históricos das classes básicas foi feita através de um gerador de contextos, onde ocorre a simulação de ações que disparam gatilhos de contexto que geram históricos e relações para os usuários, conteúdos e locais, preenchendo alguns de seus campos e servindo para outros cálculos. O gerador funciona aleatoriamente, mas com algumas variáveis direcionadas, garantindo as características desejadas nos históricos das instâncias das classes básicas.

$\mathrm{Na}$ amostra, foram inseridos dados instanciando-se as classes básicas (person, content e location) de forma que $1 / 3$ (um terço) de cada uma delas assumissem características importantes de um dos domínios. Também instanciou-se 1/3 (um terço) com instâncias sem domínio definido, totalizando assim três segmentos instanciados.

Nas simulações e nas análises dos dados em cada caso, manipularam-se as classes Person, e as escolhas de Content e Location da seguinte forma: a) Person: As instâncias desta classe foram alteradas para mostrar as características desejadas quanto à idade e ao sexo. Através dos dados do Instituto Brasileiro de Geografia e Estatística (IBGE), definiu-se as distribuições das características da classe person. Com isso, no atributo idade tivemos: Faixa de Idade: 10-24 anos; 25-39 anos; 40-54 anos; 55-69 anos e 70 acima, com os respectivos percentuais: 17,80\%; 32,51\%; 30,55\%; 21,91\% e 5,03\%; Já no atributo sexo, tivemos: masculino - 47,90\% e feminino - 52,10\%. b) Content e Location: Tiveram suas características definidas para se adequar igualmente aos domínios (segmentos) selecionados, sendo assim: Diabetes (1/3), Esclerose Lateral Amiotrófica (1/3) e ainda o segmento sem domínio definido (1/3). c) Relation: As relações RelationPersonPerson; RelationPersonContent; RelationPersonLocation $e$ RelationContentLocations foram definidas para simular todas as possibilidades de disparo de contexto com base nas proporções.

O método de avaliação de sistemas de recomendação é feito basicamente sob a satisfação do utilizador, pois tipicamente estamos preocupados em perceber o grau de aceitação das recomendações, ou seja, quantificar o número de aceitação ou rejeição dos utilizadores sobre os itens recomendados (Reis, 2012). Neste sentido, a Tabela 3 apresenta os métodos tradicionais de avaliação da recomendação, resumindo as métricas mais comuns e a Tabela 4 mostra o número de ocorrências que se enquadram em cada uma das categorias, o que servirá para calcular as respectivas métricas.

Tabela 3 - Resultados possíveis de uma recomendação (Reis, 2012) 


\begin{tabular}{|l|l|l|}
\hline & \multicolumn{1}{|c|}{ Recomendados } & \multicolumn{1}{c|}{ Não Recomendados } \\
\hline Preferidos & Verdadeiro-Positivo (VP) & Falso-Negativo (FN) \\
\hline Não Preferidos & Falso-Positivo (FP) & Verdadeiro-Negativo (VN) \\
\hline
\end{tabular}

Tabela 4 - Métricas de performance de um sistema de recomendação (Reis, 2012)

\begin{tabular}{|c|c|c|}
\hline Métrica & Expressão & Interpretação \\
\hline Precision & $\begin{array}{c}\mathrm{n}^{0} \text { de VP } \\
\mathrm{n}^{0} \text { de } V P+n^{0} \text { de FP }\end{array}$ & $\begin{array}{l}\text { Mede a probabilidade de um item } \\
\text { recomendado ser relevante. }\end{array}$ \\
\hline Recall & $\begin{array}{c}\mathrm{n}^{0} \text { de VP } \\
\mathrm{n}^{0} \text { de } V P+\mathrm{n}^{0} \text { de FN }\end{array}$ & $\begin{array}{l}\text { Mede a probabilidade de um item relevante ser } \\
\text { recomendado. }\end{array}$ \\
\hline $\begin{array}{l}\text { False } \\
\text { Positive Rate }\end{array}$ & $\begin{array}{c}\mathrm{n}^{0} \text { de FP } \\
\mathrm{n}^{0} \text { de } F P+n^{0} \text { de VN }\end{array}$ & $\begin{array}{l}\text { Mede a probabilidade de um item ser mal } \\
\text { recomendado. }\end{array}$ \\
\hline F-measure & $2 \times \frac{\text { Precision }+ \text { Recall }}{\text { Precison } \times \text { Recall }}$ & $\begin{array}{l}\text { Combina as características do Precision e do } \\
\text { Recall numa métrica conjunta. }\end{array}$ \\
\hline
\end{tabular}

\section{Experimentos e Resultados}

Os resultados finais foram analisados sobre os dois eventos citados na seção anterior, e consistiram na utilização da métrica Precision. A escolha desta métrica se deu pelas características inerentes ao sistema de recomendação, pois suas peculiaridades anulam a viabilidade da análise de Negativos, Verdadeiros ou Falsos. Devido o número de conteúdos Verdadeiros e Falsos serem bem maiores que o tamanho da recomendação final faz o número de Negativos ser alto também. Assim justifica-se a escolha da métrica Precision e o descarte de False Positive Rate e Recall.

Usando a métrica escolhida nos dois testes, obtivemos os seguintes resultados:

a) Em um contexto neutro, foi solicitado pelo sistema, recomendações para usuários que têm históricos voltados a cada um dos dois domínios instalados no sistema. Aplicando a métrica Precision com 40 recomendações, obtivemos: 721 Verdadeiro-Positivo (VP) e 119 Falso-Positivo (FP), resultando assim em um percentual de 85,83\%.

b) Em contextos voltados a cada um dos dois domínios instalados, foi solicitado pelo sistema, recomendações para usuários que têm um histórico sem domínio definido. Aplicando a métrica Precision com 40 recomendações, obtivemos: 838 VerdadeiroPositivo (VP) e 02 Falso-Positivo (FP), resultando um percentual de 99,76\%.

Outras recomendações intermediárias também foram analisadas aplicando a métrica Precision. Abaixo são apresentados os resultados:

a) Lista de Colaboradores: Com 1200 recomendações obtivemos: 1200 VerdadeiroPositivo (VP) e "zero" Falso-Positivo (FP), resultando em um percentual de $100 \%$.

b) Lista Colaborativa Ponderada: Com 40 recomendações obtivemos: 965 VerdadeiroPositivo (VP) e 235 Falso-Positivo (FP), resultando em um percentual de 80,42\%.

c) Lista Baseada em Conteúdo: Com 1200 recomendações obtivemos: 1200 VerdadeiroPositivo (VP) e "zero" Falso-Positivo (FP), resultando em um percentual de 100\%.

d) Lista Ubíqua: Com 40 recomendações obtivemos: 3365 Verdadeiro-Positivo (VP) e 235 Falso-Positivo (FP), resultando assim em um percentual de 93,47\%.

\section{Considerações Finais e Trabalhos Futuros}

Neste artigo foi apresentado um sistema de recomendação utilizado dentro de um modelo para aplicação de aprendizagem informal no contexto de Saúde 2.0. O MobiLEHealth contempla a recomendação personalizada de conteúdos de saúde, 
voltados para usuários portadores de doenças crônicas, colocando o usuário como agente ativo e responsável pelo seu tratamento e cuidados relacionados à sua saúde.

Os resultados dos testes demonstraram que é possível prover aprendizagem informal recomendando conteúdos personalizados, a partir dos interesses do usuário, por meio de perfis semânticos, relacionados a domínios de conhecimentos e gerados a partir de sua experiência e informações referentes à sua saúde, através do monitoramento do seu contexto diário.

Como trabalho futuro, pretende-se realizar um estudo de caso com usuários portadores de doenças crônicas, a fim de comprovar a eficiência e eficácia do sistema proposto no provimento de conteúdos personalizados e, principalmente, na capacidade de prover aprendizagem informal sobre saúde pessoal de forma flexível e ubíqua.

\section{Referências}

CHILDS, S. Developing health website quality assessment guidelines for the voluntary sector: outcomes from the Judge Project. Health information and libraries journal, v. 21 Suppl 2, p. 1426, 2004.

COELLO, J. M. A.; YUMING, Y.; TOBAR, C. M. A Memory-based Collaborative Filtering Algorithm for Recommending Semantic Web Services. , v. 11, n. 2, p. 795-801, 2013.

HUGHES, B.; JOSHI, I.; WAREHAM, J. Health 2.0 and Medicine 2.0: tensions and controversies in the field. Journal of Medical Internet Research, v. 10, n. 3, p. e23, 2008.

JIUGEN, Y.; RUONAN, X.; XIAOQIANG, H. Constructing informal learning mode based on social software. 6th International Conference on Computer Science \& Education (ICCSE), p. 1227-1230, IEEE, 2011.

MARTINS JÚNIOR, H. N.; COSTA, E. B.; OLIVEIRA, T. T. M.; SILVA, A. P.; BITTENCOURT, I. I. Sistema de Recomendação Híbrido para Bibliotecas Digitais que Suportam o Protocolo OAI-PMH. In: XXII Simpósio Brasileiro de Informática na Educação, SBIE, Aracaju, SE. Disponível em: <http://ceiesbc.tempsite.ws/pub/index.php/sbie/article/view/1584>. Acesso em: 2/4/2014., p. 140-149, 2011.

MENDES NETO, F. M.; COSTA, A. A. L.; SOMBRA, E. L.; MOREIRA, J. D. C.; VALENTIM, R. A. M.; SAMPER, J. J.; NASCIMENTO, R. P. C.; FLORES, C. D. Content's Personalized Recommendation for Implementing Ubiquitous Learning in Health 2.0. Revista IEEE América Latina, 2014.

MENDES NETO, F. M.; COSTA, A. A. L.; SOMBRA, E. L.; MOREIRA, J. D. C.; VALENTIM, R. A. M.; SAMPER, J. J.; NASCIMENTO, R. P. C.; FLORES, C. D. An approach for recommending personalized contents for homecare users in the context of health 2.0. 7th Euro American Association on Telematics and Information Systems - EATIS, 2014.

REIS, L. F. M. DOS. Sistema de Recomendação Baseado em Conhecimento. Dissertação de Mestrado, p. 110, 2012.

ROTHLAUF, F. Representations for Genetic and Evolutionary Algorithms, 2nd ed. Mannheim: Springer, Heidelberg, 2006.

SU, C.-J.; WU, C.-Y. JADE implemented mobile multi-agent based, distributed information platform for pervasive health care monitoring. Applied Soft Computing, v. 11, n. 1, p. 315-325, 2011.

VIEIRA, F.; NUNES, M. DICA: Sistema de Recomendação de Objetos de Aprendizagem Baseado em Conteúdo. Scientia Plena, v. 8, p. 1-10, 2012. 